\title{
CLINICAL AND MAGNETIC RESONANCE IMAGING FEATURES OF INFLAMMATORY VERSUS NEOPLASTIC MEDIAL RETROPHARYNGEAL LYMPH NODE MASS LESIONS IN DOGS AND CATS
}

\author{
Philippa J. Johnson, Richard Elders, Pascaline Pey, Ruth Dennis
}

\begin{abstract}
Medial retropharyngeal lymph node (MRLN) mass lesions are a common cause of cranial cervical masses in dogs and cats, and are predominantly due to metastatic neoplasia, primary neoplasia, or inflammatory lymphadenitis. The purpose of this retrospective cross-sectional study was to test the hypothesis that clinical and magnetic resonance imaging (MRI) characteristics for dogs and cats with MRLN mass lesions would differ for inflammatory vs. neoplastic etiologies. Dogs and cats with MRLN mass lesions that had undergone MRI and had a confirmed cytological or histopathological diagnosis were recruited from medical record archives. Clinical findings were recorded by one observer and MRI characteristics were recorded by two other observers who were unaware of clinical findings. A total of 31 patients were sampled, with 15 in the inflammatory lymphadenitis group and 16 in the neoplasia group. Patients with inflammatory lymphadenitis were more likely to be younger and present with lethargy $(P=0.001)$, pyrexia $(P=0.000)$, and neck pain $(P=0.006)$. Patients with inflammatory lymphadenitis were also more likely to have a leukocystosis $(P=0.02)$ and segmental neutrophilia $(P=\mathbf{0 . 0 0 1})$. Inflammatory masses were more likely to have moderate or marked MRI perinodal contrast enhancement $(P=0.021)$ and local muscle contrast enhancement $(P=0.03)$ whereas the neoplastic masses were more likely to have greater MRI width $(P=0.002)$ and height $(P=0.009)$. In conclusion, findings indicated that some clinical and MRI characteristics differed for dogs and cats with inflammatory vs. neoplastic medial retropharyngeal lymph node masses. Although histopathological or cytological diagnosis remains necessary for confirmation, these findings may help with the ranking of differential diagnoses of future cases. (C) 2015 American College of Veterinary Radiology.
\end{abstract}

Key words: lymphadenitis, magnetic resonance imaging, mass, medial retropharyngeal lymph node, neoplasia.

\section{Introduction}

$\mathrm{M}$ ASS LESIONS ARISING IN THE CERVICAL region are often encountered in the dog and cat. ${ }^{1}$ The medial retropharyngeal lymph node is a common origin for these mass lesions and most have a primary neoplastic, metastatic, or inflammatory aetiology. ${ }^{1,2}$ These lesions can cause significant morbidity in patients due to their mass effect and the systemic consequences of the underlying pathology involved. Differentiating between neoplastic and inflammatory etiologies for these mass lesions is important as the management and prognosis of these two disease processes is different. ${ }^{3-6}$ Identification of imaging features that can differentiate between these two conditions can help

From the Departments of Radiology (Johnson, Dennis) and Oncology (Elders) The Centre for Small Animal Studies, Animal Health Trust, and Ecole Nationale Veterinaire d'Alfort, Medical Imaging (Pey).

Previous presentations and abstracts: This study has been presented at American College of Veterinary Radiology Annual Conference in St. Luis in 2014.

Funding sources: No funding was obtained for this research.

Address correspondence and reprint requests to Philippa Johnson, at the above address. E-mail: philippa.johnson@gmail.com

Received March 24, 2014; accepted for publication June 17, 2015. doi: $10.1111 /$ vru. 12288 guide clinical decisions and may go on to be applied to mass lesions in regions that are more challenging to biopsy.

The anatomy of medial retropharyngeal lymph nodes has been extensively described. ${ }^{7,8}$ They lie in fascial fat between the wings of the atlas, mandibular salivary glands, common carotid arteries, and pharynx. They receive afferent lymph vessels from the tongue, tonsils, oral, nasal and pharyngeal cavities, salivary glands, external ear canal, larynx, esophagus, and surrounding lymph nodes. ${ }^{9}, 10$ Neoplastic enlargement of lymph nodes has been reported to occur either from primary immune system neoplasia such as lymphoma, or from lymphatic or hematogenous metastasis from primary neoplasia, most commonly arising within the afferent lymphatic drainage field. ${ }^{6,10}$ Common neoplasms that metastasize to the medial retropharyngeal lymph nodes have been reported to include tonsillar squamous cell carcinomas, mast cell tumors, thyroid carcinomas, and sarcomas and carcinomas of the maxilla, nasal cavity, and aural region. ${ }^{2,6}$ Clinical reports have indicated that patients with neoplasia primarily present for the presence of a cervical mass, and additionally with lethargy, anorexia, coughing

Vet Radiol Ultrasound, Vol. 57, No. 1, 2016, pp 24-32. 
dysphagia, Horner's syndrome, and anemia has been described possibly as a result of the primary neoplasm ${ }^{4,11,12}$ Lymphadenitis has been classified into noninfectious and infectious types. Noninfectious lymphadenitides have included autoimmune or idiopathic forms and are poorly documented in the veterinary literature. Infectious lymphadenitis has been reported secondary to bacterial (Mycobacterium spp., Pasteurella spp., Bacteriodes spp., Fusobacerium spp., Yersinia pestis, Francisella tularensis, and Bartonella spp.), fungal (systemic histoplasmosis, blastomycosis, cryptococcosis, and sporotrichosis), protozoal, algal, or metazoal infections and can have suppurative and nonsuppurative forms. ${ }^{13-15}$ Lymphadenitis has been described as a primary or secondary condition and has been associated with other diseases such as juvenile cellulitis (puppy strangles) in which diffuse lymphadenomegaly can be observed. ${ }^{5}$ Secondary lymphadenitis has been reported in response to the lymphatic drainage of inflammatory agents and/or their products. Disruptions in lymph drainage have been suggested as an underlying cause for edema, inflammation, abscessation, and granulomatous reaction. Clinical reports of dogs with lymphadenitis have described signs of lethargy and pyrexia associated with leucocytosis and visible lymph node enlargement. ${ }^{13,14,16}$

Magnetic resonance imaging (MRI) has been established as a technique for evaluating patients with cervical mass lesions. ${ }^{17-23}$ Both neoplastic and inflammatory etiologies have been reported to cause enlarged, rounded nodes with loss of the fatty hilum and cortical expansion. Additionally both can exhibit central necrosis with central poorly contrast-enhancing areas on postcontrast pulse sequences. The presence of fat stranding and reticulation of the fat adjacent to nodes have been related to cervical lymphadenitis using computed tomography. Extracapsular spread and invasion of adjacent tissues have been described as MRI features of neoplastic lesions. ${ }^{17}$ These features have been suggested as a possible way of helping to differentiate between neoplastic and inflammatory mass lesions in the neck, however to the authors knowledge no study has directly compared these lesion types. ${ }^{18}$ The MRI appearance of metastatic lymphadenopathy due to thyroid carcinoma and mast cell tumor dissemination have been documented in dogs. However, the MRI appearance of inflammatory mass lesions have not been described in dogs and there has been no study that compares the MRI features of lymph node mass lesions of neoplastic and inflammatory etiologies in dogs or cats.

Objectives of this descriptive, cross-sectional study were to compare clinical and MRI features of neoplastic and inflammatory mass lesions of the medial retropharyngeal lymph nodes in a sample of dogs and cats. We proposed a hypothesis that some clinical features and MRI characteristics of medial retropharyngeal lymph node masses would differ for infectious/inflammatory vs. neoplastic etiology groups.

\section{Materials and Methods}

This study was performed by searching the medical record archive of the Animal Health Trust for dogs or cats diagnosed with retropharyngeal lymph node mass lesions between January 2007 and July 2013. The inclusion criteria were that the patient had undergone MRI with a 1.5 T scanner (Signa Echospeed, General Electric, Milwaukee, WI), with the patients positioned in dorsal or ventral recumbency, with the relevant area in an extremity or head coil, which identified a lymph node mass lesion that measured greater than $2.0 \mathrm{~cm}$ in either the width and/or height dimension and that a histopathological or cytopathological diagnosis of neoplasia or lymphadenitis as the underlying cause for the lymph node enlargement had been made. Cases were excluded if the image quality was not diagnostic or if the field of view did not demonstrate the lesions adequately. ${ }^{6}$

One author (P.J.J.) reviewed the medical records and recorded the following data for each included patient, where available: sex, breed, age, results of histopathology and cytopathology of the mass lesions, final diagnosis, presenting/historical problem, physical examination findings, biochemical and hematological findings, results from infectious disease screening, mass culture, and any concurrent or associated disease processes.

Retrieved MRI studies were randomized and made anonymous. They were evaluated by a two European diplomates in veterinary diagnostic imaging (P.J.J. and P.P.), who was aware of the study design however were unaware of the final histopathological diagnosis. The cases were evaluated independently and when there was discrepancy in the results a consensus was reached. The images were evaluated with the use of image viewer software (OsiriX Imaging Software ${ }^{\circledR}$ osirix@osirixviewer.com) on a computer (Apple $\odot$ Cupertineo, CA) and the display settings could be adjusted as each radiologist desired. Subjective characteristics recorded for each lymph node mass included the following: lateralization (unilateral or bilateral), margination (smooth or irregular), the presence of cavitation, the loss of nodal hilus structure (Fig. 1A-C), the T2-weighted signal pattern of the masses (homogeneous or heterogeneous), and where possible the contrast-enhancement characteristics of the mass lesions (diffuse or peripheral, heterogeneous, or homogeneous). Objectively each mass lesion was measured for maximum height and width on transverse images by each observer. A width:height ratio was calculated from these two measurements. Characteristics of the perinodal fat and musculature were evaluated for contrast enhancement and grouped as none/mild or, 

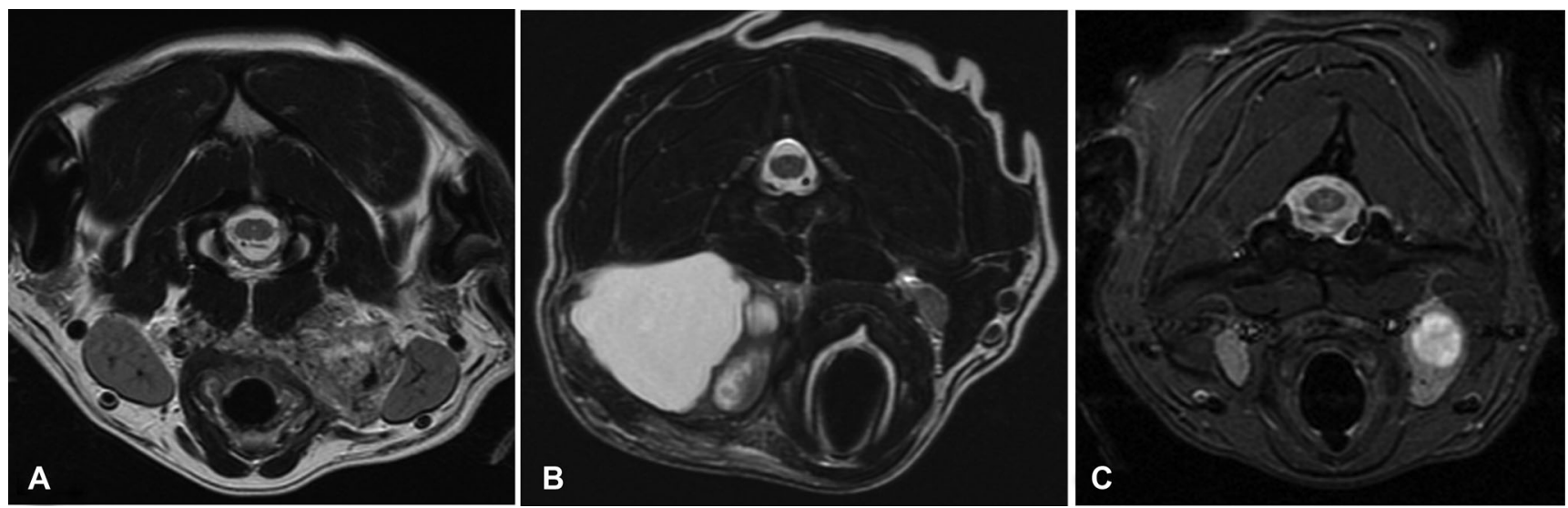

FIG. 1. T2-weighted transverse plane images demonstrating several of the structural features analyzed. (A) A 10-year-old female boxer who presented with a palpable neck mass confirmed to be undifferentiated sarcoma. This mass demonstrates the feature of an irregular margination, loss of hilus structure, and heterogeneous T2-weighted signal. (B) A 10-year-old, male Labrador who presented for dysphagia. This mass lesion was confirmed to be metastatic carcinoma and demonstrates cavitation and loss of hilus structure. (C) A 2-year-old flat-coated retriever who presented with pyrexia and confirmed to have necrosuppurative lymphadenitis. The affected left medial retropharyngeal lymph node demonstrates central T2-weighted hyperintensity and maintenance of the hilus structure.
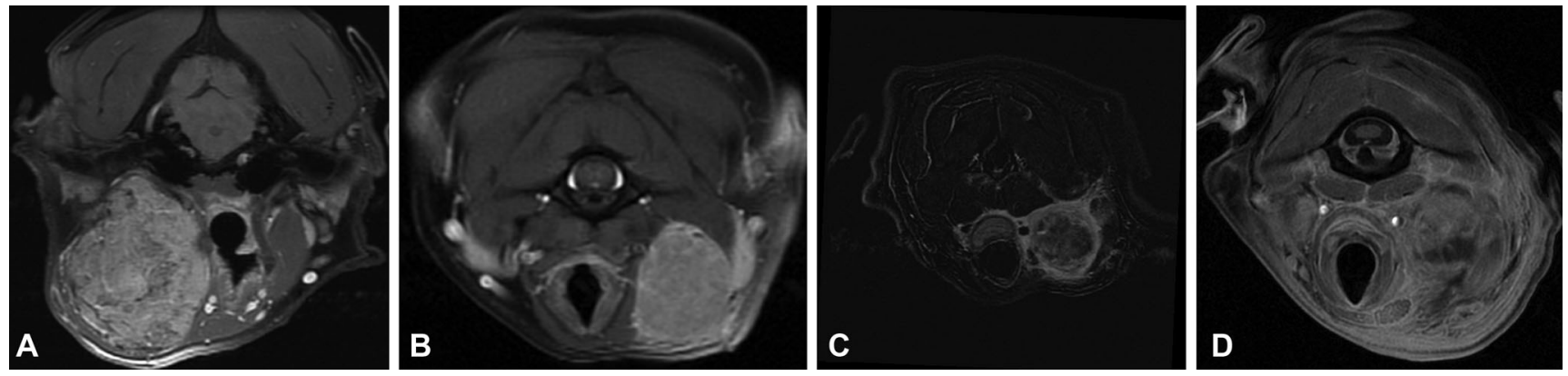

FIG. 2. Transverse plane postcontrast images of four types of laryngeal masses demonstrating the four different grades of perinodal inflammation. (A) SPGR postcontrast fat suppressed image. Spindle cell sarcoma mass lesion in a German shepherd dog who presented for an enlarging neck mass. This mass lesion is diffusely and markedly contrast enhancing however demonstrates no enhancement external to the mass lesion. Note that there is loss of fat suppression in the ventral subcutaneous fat. (B) Pre- and postcontrast subtraction image. Hodgkin's like lymphoma mass lesion in a 13-year-old male neutered domestic shorthaired cat who presented for an palpable neck mass. This image demonstrates the presence of mild perinodal inflammation involving only the tissue immediately adjacent to the mass lesion. (C) Pre- and postcontrast subtraction image. Metastatic tonsillar carcinoma mass lesion in a 7-year-old male neutered German shepherd dog presenting for dysphagia and lethargy. This image demonstrates moderate perinodal inflammation extending along fascial planes local to the mass lesion. (D) SPGR postcontrast fat suppressed image. Suppurative lymphadenitis mass lesion in a 2-year-old female spayed standard poodle who presented for pyrexia and lethargy. This image in an example of marked perinodal inflammation involving fascial planes distant to the mass lesion.

moderate/marked. Perinodal contrast enhancement was classified as none, mild when the change was confined to the fascial layer immediately adjacent to the lesion, moderate when reaction extended along local fascial planes, and marked when contrast enhancement involved distant tissues several fascial planes from the mass (Fig. 2A-D). Additionally, the presence of contrast enhancement within the local muscles was documented. Other lymph node enlargement or additional abnormal imaging findings were also noted.

Statistical tests were selected and performed by one author (P.J.J.) using statistics software (IBM SPSS Statistics ${ }^{\odot}$, release 22.0.0.0, 64-bit edition, IBM Corporation and others 1989, 2013). Dogs were assigned to inflammatory or neoplastic groups based on histopathologic or cytologic diagnosis in the medical record. The prevalence of specific clinical and MRI features was calculated within each etiological group (neoplastic vs. inflammatory). A correlation between the two observers for the mass dimension measurements was performed using the Pearson's correlation. The mean of the two measurements between the observers was calculated. The age, average height, and average width measurement data were identified as being not normally distributed via the Shapiro-Wilk's test $(P>0.5)$ and a visual inspection of their histograms, normal Q-Q plots, and box plots. Statistical difference between the two groups was therefore calculated using a nonparametric Mann-Whitney $U$ test with demonstration of an exact $P$-value. A $P$-value threshold of 0.05 was used to signify statistical significance. Receiver operator curves (ROC) were formed for height, width, and age to identify ability for these measures for group prediction. Cut-off 
thresholds were calculated when a factor was considered a good or excellent test (area under the curve (AUC) 0.8-1.0). For the clinical factors and the subjective MRI characteristics, statistical difference was tested using the Fischer's exact test $(P<0.05)$. When a clinical or MRI feature was significantly more likely to occur in one group then positive and negative predictive values (PPV, NPV) were calculated.

\section{Results}

\section{Clinical Findings}

Thirty-one cases fitted the inclusion criteria. Of the 31 cases an inflammatory, nonneoplastic diagnosis was obtained with histology in 10 cases and cytology in five cases. A neoplastic diagnosis was reached via histology in 13 cases and cytology in three cases. The nonneoplastic group $(n=15)$ included $14 \mathrm{dogs}$ and one cat and the final diagnoses included suppurative lymphadenitis $(n=7 \mathrm{dogs}$, $46.7 \%)$, pyogranulomatous lymphadenitis $(n=2$ dogs, 1 cat, 20\%), unspecified lymphadenitis $(n=3$ dogs, $20 \%)$, granulomatous lymphadenitis ( $n=1 \mathrm{dog}, 6.6 \%)$ and hemorrhagic lymphadenitis $(n=1 \mathrm{dog}, 6.6 \%)$. This group was composed of nine females and six males. The neoplastic group $(n=16)$ included 12 dogs and four cats and the final diagnoses included metastatic tonsillar carcinoma $(n=5$ dogs, $31.2 \%)$, lymphoma $(n=3$ cats $18.8 \%)$, metastatic thyroid carcinoma $(n=3 \mathrm{dogs}, 18.8 \%)$, poorly differentiated sarcoma $(n=1 \mathrm{dog}, 1$ cat, $12.5 \%)$, histiocytic sarcoma $(n=2$ dogs, $12.5 \%)$, and spindle cell sarcoma $(n=$ $1 \mathrm{dogs}, 6.3 \%)$. This group was composed of seven females and nine males. Both etiological groups contained a variety of different dog and cat breeds. There was a statistical difference in the mean ages of the etiological groups $(P=$ $0.001)$; in the inflammatory group the mean age was 3.8 years $( \pm 3.17)$ whereas in the neoplastic group the mean age was 8.6 years $( \pm 3.53)$. An ROC identified that the age of the patient was a good test for predicting whether the mass was either an inflammatory or neoplastic with an AUC of 0.848 (Fig. 3A). Threshold coordinates optimizing both sensitivity and specificity identified an age less than 6.5 years as having a sensitivity of $80 \%$ and a specificity of $75 \%$ for prediction of the inflammatory group.

The clinical signs and physical examination findings for each etiological group are summarized in Table 1 . When assessed statistically it was found that significantly more patients bearing inflammatory lesions presented with lethargy $(P=0.001)$. The presence of lethargy in the clinical history has a positive predictive value of $80.0 \%$ and negative predictive value of $81.2 \%$ for the diagnosis of an inflammatory etiology. The most common physical examination findings in the inflammatory group $(n=15)$ were the presence of pyrexia $(n=12,80 \%)$ and pain on palpation or movement of the neck $(n=9,60 \%)$. When the clinical findings were assessed statistically it was found that significantly more patients bearing inflammatory lesions presented with pyrexia $(P=0.000)$ and neck pain $(P=0.006)$. Pyrexia had a positive predictive value of $100.0 \%$ and a negative productive value of $80.0 \%$, where as neck pain had a PPV of $81.8 \%$ and an NPV of $70.0 \%$ for the diagnosis of an inflammatory etiology.

The most frequently recorded clinicopathological abnormalities of both etiological groups are presented in Table 2 . Leucocystosis was statistically more often present in the inflammatory group $(P=0.020)$. It has a PPV of 100.0 and a NPV of 66.7. Additionally, a segmental neutrophilia was statistically more likely in the inflammatory group $(P=0.001)$. The presence of neutrophilia on blood work had a PPV $100 \%$ and an NPV of $73.9 \%$ for a diagnosis of an inflammatory etiology. Based on clinical suspicions, individual patients were screened for a number of infectious diseases including Toxoplasma gondii, Neospora caninum, canine distemper virus, Mycobacterium spp., Borrelia spp., Ehrilichia spp., Bartonella spp., and feline immunodeficiency virus, feline leukemia virus, and feline coronavirus. All tests for infectious diseases in both etiological groups were negative. Culture of the masses was performed in 12 patients in the inflammatory group $(n=15): 11$ samples revealed no fungal or aerobic or anaerobic bacterial growth and one sample had a positive growth of coagulase negative Staphylococcus spp. Five neoplastic masses $(n=16)$ were sampled for culture and all showed negative growth. In the inflammatory group concurrent illnesses identified included tonsillitis $(n=3,20 \%)$, rhinitis $(n=1,7.6 \%)$, and spindle cell neoplasia on the elbow $(n=1,7.6 \%)$. In the neoplastic group concurrent illnesses included tonsillar carcinoma ( $n=5,29.4 \%)$, multicentric or disseminated lymphoma $(n=1,6.3 \%)$, thyroid carcinoma $(n=3,17.6 \%)$, glossopharyngeal neuropathy ( $n=1,5.8 \%)$, locoregional lymphoma $(n=15.8 \%)$, intracranial extra-axial mass lesion $(n=1$, $5.8 \%)$, humeral osteoma $(n=1,5.8 \%)$, disseminated histiocytic sarcoma $(n=1,5.8 \%)$, bronchitis $(n=1,5.8 \%)$, sebaceous adenitis $(n=1,5.8 \%)$, and mild myxomatous degeneration of the mitral valve $(n=1,5.8 \%)$.

\section{MRI Findings}

The MRI studies that were evaluated consisted of T2-weighted, T1-weighted, and gradient echo sequences. T2-weighted (T2-W) fast spin echo (FSE), with or without fat suppression (TR 2,520-5,060, TE 82.4-89.8) or short tau inversion recovery (STIR; TR 3,420, TE 24 26.1) sequences were available for review. For contrastenhancement evaluation, 28 cases had T1W (TR 440 800, TE 10.2-16.5) before and after intravenous bolus administration of gadopentetate dimeglumine at $0.1 \mathrm{~mL} / \mathrm{kg}$ 


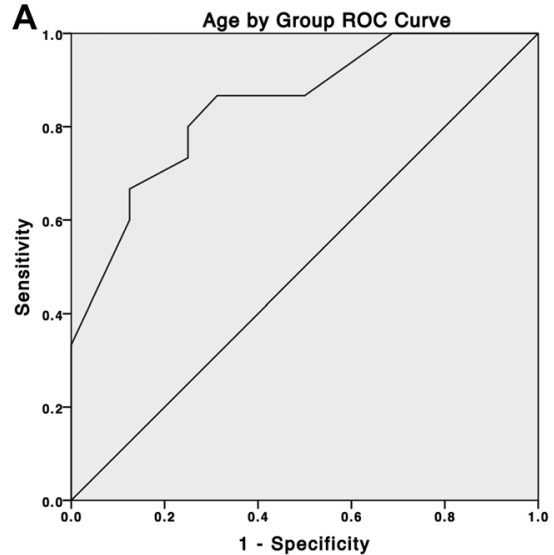

Diagonal segments are produced by ties.

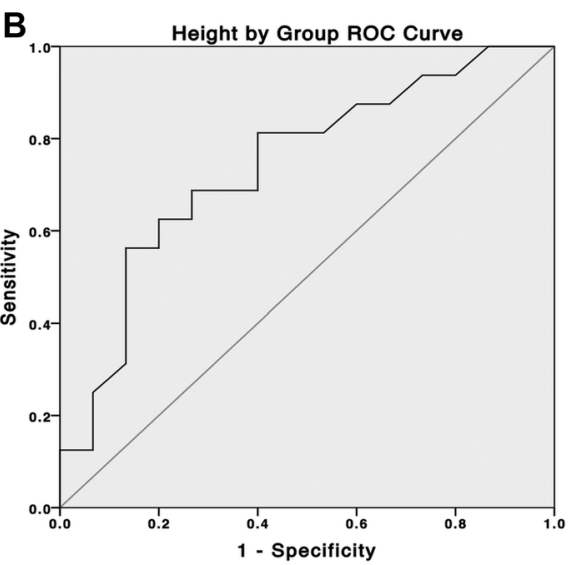

Diagonal segments are produced by ties.

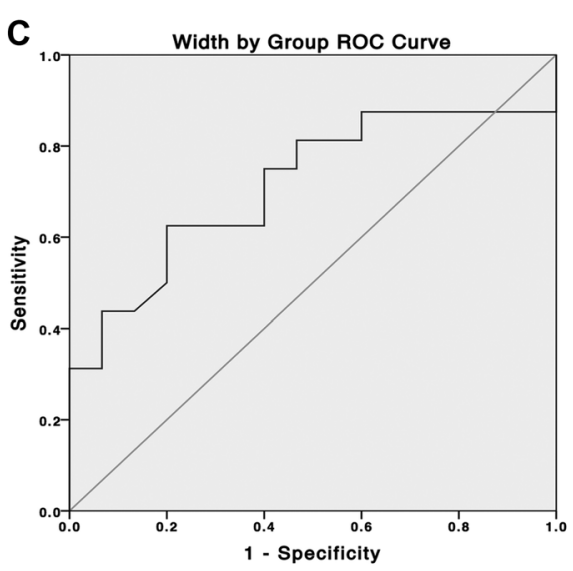

Diagonal segments are produced by ties.

FIG. 3. The receiver operator curves (ROCs) that were formed for height, width, and age to identify ability for these measures for group prediction. Cut-off thresholds were calculated when a factor was considered a good or excellent test (area under the curve (AUC) 0.8-1.0). (A) Age by group ROC. Identified that the age of the patient was a good test for predicting whether the mass was either an inflammatory or neoplastic with an AUC of 0.848. Threshold coordinates optimizing both sensitivity and specificity identified an age less than 6.5 years as having a sensitivity of $80 \%$ and a specificity of $75 \%$ for prediction of the inflammatory group. (B) Height by group ROC. Identified height as a fair test for differentiation between groups with an AUC of 0.742 . (C) Width by group ROC. Identified the width measure to be a fair test for differentiation with an AUC of 0.715 .

TABle 1. Presenting Clinical Signs and Examination Findings Identified in the Inflammatory and Neoplastic Groups of 31 Dogs and Cats Presented for Neck Masses*

\begin{tabular}{|c|c|c|c|c|c|c|}
\hline \multicolumn{7}{|c|}{ Clinical signs and physical examination findings } \\
\hline Clinical signs & Inflammatory $(n=15)$ & Neoplastic $(n=16)$ & & $P$-value & PPV & NPV \\
\hline Neck swelling/mass & $14(93.3 \%)$ & $15(93.8 \%)$ & & 1.000 & & \\
\hline Lethargy & $12(80.0 \%)$ & $3(18.8 \%)$ & & 0.001 & 80.0 & 81.25 \\
\hline Inappetance & $6(40.0 \%)$ & $2(12.5 \%)$ & & 0.113 & & \\
\hline Dysphagia & $1(6.6 \%)$ & $4(25.0 \%)$ & & 0.333 & & \\
\hline Voice alteration or dysphonia & $2(13.3 \%)$ & $0(0.0 \%)$ & & - & & \\
\hline Ocular discharge & $1(6.6 \%)$ & $0(0.0 \%)$ & & - & & \\
\hline Upper respiratory noise (stridor/stertor) & $0(0.0 \%)$ & $3(18.7 \%)$ & & - & & \\
\hline Cranial nerve deficits & $0(0.0 \%)$ & $1(6.3 \%)$ & & - & & \\
\hline Horner's syndrome & $0(0.0 \%)$ & $1(6.3 \%)$ & & - & & \\
\hline Balance disturbance (dysequilibrium) & $0(0.0 \%)$ & $1(6.3 \%)$ & & - & & \\
\hline \multicolumn{7}{|l|}{ Physical examination } \\
\hline Pyrexia & $11(73.3 \%)$ & $0(0.0 \%)$ & 0.000 & & 100.00 & 80.00 \\
\hline Pain & $9(60.0 \%)$ & $2(12.5 \%)$ & 0.006 & & 81.81 & 70.00 \\
\hline Enlarged tonsils & $2(13.3 \%)$ & $5(31.3 \%)$ & 0.394 & & & \\
\hline Enlarged mandibular/superf cervical LNs & $5(33.3 \%)$ & $2(12.5 \%)$ & 0.220 & & & \\
\hline Trismus & $1(6.6 \%)$ & $0(0.0 \%)$ & - & & & \\
\hline
\end{tabular}

* Statistical significance was calculated in all clinical signs that were demonstrated in less than five cases. $P$-values were calculated using Pearson Chi-Square test or Fischer's exact test when numbers were small. The results with statistically significant differences between groups are highlighted in bold $(P$-value $<0.05)$.

of bodyweight (Multi-Hance, Bracco Imaging SpA or Gadovist, Bayer Schering Pharma). Some postcontrast T1weighted sequences were fat suppressed and pre- and postcontrast subtraction images were constructed routinely in the non-fat suppressed sequences. Ten cases had T $2 *$ gradient echo sequences available (TR 440-800, TE 15) and six had the 3D sequence spoiled gradient echo (SPGR) (TR 930, TE 3.7). All studies were obtained in transverse planes (field of view $12-20 \mathrm{~cm}$, slice thickness 3-7 mm) with dorsal and/or sagittal planes available in some cases.

An overview of the MRI findings in both etiological groups is shown in Table 3 . When the lymph nodes were evaluated none of the subjective $\mathrm{T} 2 \mathrm{~W}$ and precontrast MRI features analyzed were statistically more significant in either group. On the objective measurements in the dog population the inflammatory masses $(n=14)$ had a mean width of $20 \mathrm{~mm}( \pm 7.0)$ and mean height of $29 \mathrm{~mm}( \pm 9.7)$ whereas the neoplastic masses $(n=12)$ had a mean width of $35 \mathrm{~mm}$ $( \pm 14.0)$ and a mean height of $42 \mathrm{~mm}( \pm 15.4)$. The neoplastic masses had statistically larger width $(P=0.002)$ and height $(P=0.009)$ measurements than the inflammatory masses. There was no statistical difference of the width/height ratio between the two groups $(P=0.190)$. These findings are summarized in Table 4 . ROC statistics 
TABLE 2. Clinicopathological Abnormalities Identified within the Two Etiological Groups of 31 Dogs and Cats with Neck Masses*

\begin{tabular}{|c|c|c|c|c|c|c|}
\hline \multicolumn{7}{|c|}{ Clinicopathological changes observed during blood analysis } \\
\hline Clinicopathological abnormalities & & Inflammatory lesions $(n=15)$ & Neoplastic lesions $(n=16)$ & $P$-value & PPV & NPV \\
\hline Red blood cells $\left(5.5-10.0 \times 10^{12} / 1\right)$ & Anemia & $4(26.6 \%)$ & $1(6.3 \%)$ & 0.172 & & \\
\hline \multirow[t]{2}{*}{ White blood cells $\left(5.5-16.9 \times 10^{9} / 1\right)$} & Leucocytosis & $7(46.7 \%)$ & $0(0 \%)$ & 0.020 & 100.00 & 66.67 \\
\hline & Leucopenia & $2(13.3 \%)$ & $2(12.5 \%)$ & - & & \\
\hline Neutrophils $\left(2.0-12.0 \times 10^{9} / 1\right)$ & Neutrophilia & $8(53.3 \%)$ & $0(0.0 \%)$ & 0.001 & 100.00 & 69.57 \\
\hline Monocytes $\left(0.3-2.0 \times 10^{9} / 1\right)$ & Monocytosis & $3(20.0 \%)$ & $1(5.8 \%)$ & - & & \\
\hline \multirow[t]{2}{*}{ Eosinophils $\left(0.1-1.49 \times 10^{9} / 1\right)$} & Eosinophilia & $3(20.0 \%)$ & $1(6.3 \%)$ & - & & \\
\hline & Eosinopenia & $4(26.7 \%)$ & $0(0 \%)$ & - & & \\
\hline Serum total protein $(55.0-75.0 \mathrm{~g} / \mathrm{l})$ & High & $2(13.3 \%)$ & $3(18.8 \%)$ & 1.000 & & \\
\hline \multirow[t]{2}{*}{ Cholesterol $(1.9-5.0 \mathrm{mmo} / \mathrm{l})$} & High & $4(26.7 \%)$ & $2(12.5 \%)$ & 0.394 & & \\
\hline & Low & $2(13.3 \%)$ & $0(0.0 \%)$ & - & & \\
\hline \multirow[t]{2}{*}{ Amylase $(900-3,000 \mathrm{U} / \mathrm{l})$} & High & $2(13.3 \%)$ & $1(6.3 \%)$ & 0.600 & & \\
\hline & Low & $2(13.3 \%)$ & $2(12.5 \%)$ & 1.000 & & \\
\hline Alkaline phosphatase (0.0-160 IU/1) & High & $4(26.6 \%)$ & $1(6.3 \%)$ & 1.720 & & \\
\hline Alanine aminotransferase $0.0-100.0 \mathrm{IU} / \mathrm{l}$ ) & High & $0(0.0 \%)$ & $3(18.8 \%)$ & - & & \\
\hline Creatinine kinase (21.0-56.0 IU/1) & High & $2(13.3 \%)$ & $4(25.0 \%)$ & 0.654 & & \\
\hline
\end{tabular}

*Statistical significance was calculated in all clinical signs that were demonstrated in less than cases. $P$-values were calculated using Pearson Chi-Square test or Fischer's exact test when numbers were small. The results with statistically significant differences between groups are highlighted in bold $(P$-value $<0.05$ ).

TABLE 3. MRI Qualitative Characteristics Identified on T2-Weighted and T1-Weighted Pre/Postcontrast Images for 31 Dogs and Cats with Neck Masses*

\begin{tabular}{|c|c|c|c|c|c|c|}
\hline \multicolumn{7}{|c|}{ Structural and contrast enhancement magnetic resonance imaging features of the mass lesions } \\
\hline \multicolumn{2}{|l|}{ MRI features } & Inflammatory lesions $(n=15)$ & \multirow{2}{*}{$\frac{\text { Neoplastic lesions }(n=16)}{12(75.0 \%)}$} & $P$-value & \multirow[t]{3}{*}{ PPV } & NPV \\
\hline \multirow[t]{2}{*}{ Masses } & Unilateral & $12(80.0 \%)$ & & 1.00 & & \\
\hline & Bilateral & $3(2.0 \%)$ & $4(25.0 \%)$ & & & \\
\hline \multirow[t]{2}{*}{ Margination } & Smooth & $6(40.0 \%)$ & $12(75.0 \%)$ & 0.48 & & \\
\hline & Irregular & $9(60.0 \%)$ & $4(25.0 \%)$ & & & \\
\hline \multirow[t]{2}{*}{ Cavitation } & Present & $6(40.0 \%)$ & $5(31.3 \%)$ & 0.611 & & \\
\hline & Not present & $9(60.0 \%)$ & $11(68.8 \%)$ & & & \\
\hline \multirow[t]{2}{*}{ Hilar structure } & Maintained & $3(20.0 \%)$ & $1(6.3 \%)$ & 0.333 & & \\
\hline & Lost & $12(80.0 \%)$ & $15(93.8 \%)$ & & & \\
\hline \multirow[t]{2}{*}{ T2-weighted signal pattern } & Heterogeneous & $10(66.7 \%)$ & $12(75.0 \%)$ & 0.704 & & \\
\hline & Homogeneous & $5(33.3 \%)$ & $4(25.0 \%)$ & & & \\
\hline \multicolumn{2}{|l|}{ Contrast enhancement features } & Inflammatory lesions $(n=13)$ & Neoplastic lesions $(n$ & & & \\
\hline \multirow[t]{2}{*}{ Mass enhancement } & Peripheral & $2(15.4 \%)$ & $3(20.0 \%)$ & 1.000 & & \\
\hline & Diffuse & $11(84.6 \%)$ & $12(80.0 \%)$ & & & \\
\hline \multirow[t]{2}{*}{ Mass enhancement pattern } & Heterogeneous & $12(92.3 \%)$ & $13(86.7 \%)$ & 1.000 & & \\
\hline & Homogeneous & $1(7.7 \%)$ & $2(13.3 \%)$ & & & \\
\hline \multirow[t]{2}{*}{ Perinodal soft tissue enhancement } & None/mild & $3(23.1 \%)$ & $10(66.7 \%)$ & & & \\
\hline & Moderate/marked & $10(76.9 \%)$ & $5(33.3 \%)$ & 0.021 & 66.67 & 68.75 \\
\hline \multirow[t]{2}{*}{ Muscle enhancement } & Present & $10(76.9 \%)$ & $3(20.0 \%)$ & 0.03 & 76.92 & 72.22 \\
\hline & Absent & $3(23.1 \%)$ & $12(80.0 \%)$ & & & \\
\hline
\end{tabular}

* Statistical significance was calculated in all clinical signs that were demonstrated in less than five cases. $P$-values were calculated using Pearson Chi-Square test or Fischer's exact test when numbers were small. The results with statistically significant differences between groups are highlighted in bold $(P$-value $<0.05)$.

were applied to the height and weight measurements and these parameters were found to be only a fair test for differentiation between the two groups (height AUC 0.742, width AUC 0.715) (Fig. 3B and C).

On evaluation of the perinodal tissues, there was an inflammatory group statistically more likely to have moderate or marked perinodal contrast enhancement $(P=0.02)$ and muscle enhancement $(P=0.03)$ than the neoplastic group. Moderate-to-marked perinodal contrast enhancement was present in $76.9 \%(n=10)$ of the inflammatory cases and only present in $33.3 \%(n=5)$ of neoplastic cases. The presence of moderate-to-marked perinodal contrast has a PPV of 66.67 and an NPV of 68.75 for the detection of an inflammatory etiology. Muscle enhancement was observed in $76.9 \%(n=10)$ of inflammatory cases and in three of the neoplastic cases $(20.0 \%)$. The presence of muscle contrast enhancement has a PPV of 76.92 and an NPV of 72.22 for the detection of an inflammatory etiology. The muscles involved were all local to the affected lymph node and included the omotransversarius m., obliquis capitis caudalis $m$., longus capitis m., rectus capitis dorsalis major m., arytenoideus transversus $m$., vocalis $m$., sternothyroideus $m$., sternocephalicus m., sternomastoideus m., sternohyoideus $m$., masseter $m$. and digastricus $m$. 
TABLE 4. MRI Measurements of Neck Masses for 31 Dogs and Cats*

\begin{tabular}{lcccc}
\hline \multicolumn{2}{l}{ Quantitative measurement results } & Inflammatory lesions/mm $(n=14)$ & Neoplastic lesions/mm $(n=12)$ & $P$-value \\
& & $29.25( \pm 9.65)$ & $42.29( \pm 15.43)$ & $\mathbf{0 . 0 0 9}$ \\
\hline Dog & Height & $19.82( \pm 6.99)$ & $35.04( \pm 13.98)$ & $0.82( \pm 0.12)$ \\
& Width & $0.68( \pm 0.11)$ & 0.190 \\
\hline & Width/height ratio & Inflammatory lesions/mm $(n=1)$ & Neoplastic lesions/mm $(n=4)$ & $P$-value \\
\hline Cat & & 27 & $23.75( \pm 6.84)$ & NA \\
& Height & 22 & $17.75( \pm 7.51)$ & NA \\
& Width & 0.89 & $0.73( \pm 0.15)$ & NA \\
\hline
\end{tabular}

*The statistical significance obtained from the Mann-Whitney $U$ test is demonstrated by an exact $P$-value, where a $P$-value $<0.05$ indicates a significant difference and is highlighted in bold.

Enlargement of other lymph nodes within the head and neck was more common in the inflammatory group with enlargement of both the contralateral medial retropharyngeal lymph node and ipsilateral or both mandibular lymph nodes present in $12(80 \%)$ cases. However, there was a wider range of lymph nodes enlarged in the neoplastic group, including the contralateral medial retropharyngeal lymph node $(n=4,25.0 \%)$, ipsilateral mandibular lymph nodes $(n=8,50.0 \%)$, ipsilateral deep cervical lymph nodes $(n=$ $1,6.6 \%$ ), and ipsilateral superficial cervical lymph nodes $(n=1,6.6 \%)$.

Tonsillar enlargement was identified on the MR images in three cases in the inflammatory group and in five cases in the neoplastic group. Additional lesions identified in the images in the inflammatory group included mild nasal turbinate thickening $(n=2,13.3 \%)$ and in the neoplastic group included thyroid mass $(n=3,17.6 \%)$, extra-axial brain mass $(n=1,6.6 \%)$, and nasopharyngeal mass $(n=$ $1,6.6 \%)$.

\section{Discussion}

The results of this study are consistent with the proposed hypothesis that the clinical and MRI features of medial retropharyngeal lymph node masses differ between inflammatory and neoplastic etiologies. There were significant differences in the signalment, presenting signs, hematological analyses results, and MRI features between dogs and cats with inflammatory vs. neoplastic mass lesions.

When comparing the signalment and history of the two groups the inflammatory group presented at a younger mean age than the neoplastic group. An age of 6.5 years was identified as being the most sensitive and specific cut off from the receiver operating curve statistics. This finding is consistent with the veterinary literature in which neoplasia is associated with an older age demographic and lymphadenitis is mostly reported in young dogs. ${ }^{4,16,24}$ Similarly, in human medicine, cervical lymphadenitis is described predominantly in children and young adults. ${ }^{15,19,25}$ The historical finding of lethargy was significantly more likely to be present in the nonneoplastic inflammatory group than the neoplastic group. This is consistent with the literature that report tonsillar squamous cell carcinoma-bearing patients infrequently present with lethargy or anorexia, although when present, these signs are associated with a significantly shorter survival time and a poorer outcome. ${ }^{4}$ Dysphagia has also been reported in these patients. ${ }^{4}$ In our study four cases with neoplastic masses presented with dysphagia may have been due to the mass effect compressing on the oropharyngeal region or from invasion of hypoglossal nerve, which runs along the medial aspect of the medial retropharyngeal lymph node and provides motor innervation to the tongue for participation in the oral and pharyngeal phases of swallowing. ${ }^{16}$

On physical examination in this study lymphadenitis cases were statistically more likely to be pyrexic and to demonstrate neck pain. These clinical signs have been described widely in relation to cranial cervical lymphadenitis both in the human and veterinary literature and have been related to the extension of inflammation outside of the lymph node capsule, and the elaboration of inflammatory cytokines. ${ }^{16,25,26}$ An underlying cause for the lymphadenitis lesions was not identified in the majority of cases and they were therefore presumed to be immune mediated. Despite 12 of 16 of the lymphadenitis lesions being cultured only one yielded bacterial growth. This might be due to the administration of antibiotics prior to presentation of the culture-negative cases. In children, the most common cause for lymphadenitis is an acute bacterial infection including mycobacterial infection extending from the oropharynx or elsewhere. ${ }^{15,27}$ In dogs, lymphadenitis has been associated with Bartonella henselae, Talaromyces helicus, mycobacterial infections, and juvenile cellulitis (puppy strangles). ${ }^{3,13,14,24,28}$

Upon assessment of the mass lesions and perinodal tissues significant differences between the two groups were identified. The size of normal medial retropharyngeal lymph nodes has been assessed with MRI in dogs and described as having an approximate width of $5-10 \mathrm{~mm}$ and a height of $10 \mathrm{~mm} \cdot{ }^{8,29,30}$ The width and height dimensions of the mass lesions were significantly larger in the neoplastic group in our study however the height:width ratio was not 
statistically different between the neoplastic and inflammatory groups. The ultrasonographic dimensions of lymph nodes have been assessed for their ability to differentiate neoplastic from nonneoplastic nodes in dogs. Similar to our findings neoplastic lymph nodes were identified as being larger when compared with nonneoplastic (hyperplastic or inflammatory) lymphadenomegaly. ${ }^{31}$ Despite these findings, it is important to bear in mind that in both the medical and veterinary literature it is reported that size is not a reliable marker for neoplastic infiltration, as small nodes can harbor small metastases. ${ }^{32-35}$ The presence of perinodal enhancement and muscle contrast enhancement was significantly more likely to be associated with an inflammatory rather than a neoplastic etiology. The high presence of perinodal contrast enhancement and inflammation around inflammatory lymph nodes is consistent with those findings described in computed tomographic studies of lymphadenitis lesions and lymph node abscessation where fat stranding, reticulation, and peripheral contrast enhancement is described. ${ }^{18,36}$

There are several imaging features documented in the literature that are suggested to indicate the presence of lymph node metastasis that were not identified as being significantly more likely in the neoplastic group over the inflammatory group in our study. These features include the loss of nodal hilar structure, the presence of central necrosis or cavitation, and irregular margination due to capsular invasion. ${ }^{35,37}$ This disparity is likely due to the fact that these studies compared reactive lymph nodes to early-stage metastasis, which is a very different cohort of pathologies from our study. Our inclusion criteria required patients to have mass lesions and were therefore at a late stage of metastasis or had an advanced inflammatory lesion. As a result of this chronicity these late-stage lesions exhibited destruction of hilus structure, capsular invasion, and central necrosis to a similar degree.
One limitation of this study is its retrospective nature. This meant that the clinical approach was not consistent between cases and the clinical findings were reliant on what was documented in the case notes rather than a standardized assessment. Additionally, the imaging protocols were not standardized for all patients and this resulted in several studies not having a postcontrast series. This study only assessed T2/T1-weighted imaging features and contrast enhancement, however in medical imaging the use of diffusion weighted (DW) MRI has been utilized to help differentiate between lymphadenitis and malignant retropharyngeal mass lesions. It was identified that signal intensity ratios on DW MRI was found to be higher and apparent diffusion coefficient values lower in suppurative lymphadenitis when compared to malignancies. ${ }^{38}$ Further investigation into these techniques in veterinary patients to assess its utility in the differentiation of these conditions could be an avenue to examine the future.

In conclusion, the proposed hypothesis was accepted with several specific clinical and MRI features being statistically more likely to occur in either the inflammatory or neoplastic groups. The statistically significant clinical features that were more likely with a lymphadenitis diagnosis included a young age at presentation, the presence of historic lethargy, clinical pyrexia, leucocytosis and segmental neutrophilia, and MRI features of a moderate or marked perinodal contrast enhancement, and the presence of muscle contrast enhancement. Features that were statistically more common in neoplastic lesions included an older patient presenting for a nonpainful mass lesion and having a significantly larger mass lesion on MRI. When evaluating MR images of medial retropharyngeal lymph node mass lesions, these clinical and imaging features might help in the ranking of differential diagnoses and provide clinical guidance toward a more likely underlying cause.

\section{REFERENCES}

1. Wisner ER, Nyland TG, Mattoon JS. Ultrasonographic examination of cervical masses in the dog and cat. Vet Radiol Ultrasound 1994;35: $310-315$.

2. Withrow SJ, Liptak JM. Oral tumors. In: Withrow and MacEwen's small animal clinical oncology, 4th ed. Missouri: Saunders Elsevier, 2007;455-475.

3. Neuber AE, vanden Broek AH, Brownstein D, et al. Dermatitis and lymphadenitis resembling juvenile cellulitis in a four-year-old dog. J Small Anim Pract 2004;45:254-258.

4. Mas A, Blackwood L, Cripps P, et al. Canine tonsillar squamous cell carcinoma - a multi-centre retrospective review of 44 clinical cases. J Small Anim Pract 2011;52:359-364.

5. Reimann KA, Evans MG, Chalifoux LV, et al. Clinicopathologic characterization of canine juvenile cellulitis. Vet Pathol 1989;26: 499-504.

6. Herring ES, Smith MM, Robertson JL. Lymph node staging of oral and maxillofacial neoplasms in 31 dogs and cats. J Vet Dent 2002; 19: $122-126$.
7. Nemanic S, Nelson NC. Ultrasonography and noncontrast computed tomography of medial retropharyngeal lymph nodes in healthy cats. Am J Vet Res 2012;73:1377-1385.

8. Kneissl S, Probst A. Magnetic resonance imaging features of presumed normal head and neck lymph nodes in dogs. Vet Radiol Ultrasound 2006;47:538-541.

9. Bezuidenhout AJ. The lymphatic system. In: Evans (ed): Miller's anatomy of the dog, Vol., 4th ed. Philadelphia: W.B. Saunders, 2013;543.

10. Belz GT, Heath TJ. Lymph pathways of the medial retropharyngeal lymph node in dogs. J Anat 1995;186(Pt 3):517-526.

11. Taeymans O, Penninck DG, Peters RM. Comparison between clinical, ultrasound, CT, MRI, and pathology findings in dogs presented for suspected thyroid carcinoma. Vet Radiol Ultrasound 2012;54:61-70.

12. Elliott JW, Cripps P, Blackwood L, et al. Canine oral mucosal mast cell tumours. Vet Comp Oncol 2013. doi: 10.1111/vco.12071.

13. Pappalardo BL, Brown T, Gookin JL, et al. Granulomatous disease associated with Bartonella infection in 2 dogs. J Vet Intern Med 2000;14: $37-42$. 
14. Tomlinson JK, Cooley AJ, Zhang S, et al. Granulomatous lymphadenitis caused by Talaromyces helicus in a Labrador Retriever. Vet Clin Pathol 2011;40:553-557.

15. Beiler HA, Eckstein TM, Roth H, et al. Specific and nonspecific lymphadenitis in childhood: etiology, diagnosis, and therapy. Pediatr Surg Int 1997; 12:108-112.

16. Dandrieux JR, Timm K, Roosje PJ, et al. Unusual systemic signs in a dog with sterile neutrophilic-macrophagic lymphadenitis and nodular panniculitis. J Am Anim Hosp Assoc 2011;47:117-121.

17. Bryson TC, Shah GV, Srinivasan A, et al. Cervical lymph node evaluation and diagnosis. Otolaryngol Clin North Am 2012;45:1363-1383.

18. McKellop JA, Bou-Assaly W, Mukherji SK. Emergency head \& neck imaging: infections and inflammatory processes. Neuroimaging Clin N Am 2010;20:651-661.

19. Gonzalez-Beicos A, Nunez D. Imaging of acute head and neck infections. Radiol Clin North Am 2012;50:73-83.

20. Faruqui S, Palacios E, Friedlander P, et al. Nontraumatic retropharyngeal abscess complicated by cervical osteomyelitis and epidural abscess in post-Katrina New Orleans: four cases. Ear Nose Throat J 2009;88:E14.

21. Pokorny E, Hecht S, Sura PA, et al. Magnetic resonance imaging of canine mast cell tumors. Vet Radiol Ultrasound 53:167-173.

22. Taeymans O, Penninck DG, Peters RM. Comparison between clinical, ultrasound, CT, MRI, and pathology findings in dogs presented for suspected thyroid carcinoma. Vet Radiol Ultrasound 2013;54:61-70.

23. Ballegeer EA, Adams WM, Dubielzig RR, et al. Computed tomography characteristics of canine tracheobronchial lymph node metastasis. Vet Radiol Ultrasound 2010;51:397-403.

24. Campora L, Corazza M, Zullino C, et al. Mycobacterium avium subspecies hominissuis disseminated infection in a Basset Hound dog. J Vet Diagn Invest 2011;23:1083-1087.

25. Imamura M, Ueno H, Matsuura A, et al. An ultrastructural study of subacute necrotizing lymphadenitis. Am J Pathol 1982;107:292-299.

26. Fraser IP. Suppurative lymphadenitis. Curr Infect Dis Rep 2009; 11:383-388.
27. Harris $\mathrm{BH}$, Webb HW, Wilkinson AH Jr., et al. Mycobacterial lymphadenitis. J Pediatr Surg 1982;17:589-590.

28. Morales SC, Breitschwerdt EB, Washabau RJ, et al. Detection of Bartonella henselae DNA in two dogs with pyogranulomatous lymphadenitis. J Am Vet Med Assoc 2007;230:681-685.

29. Kneissl S, Probst A. Comparison of computed tomographic images of normal cranial and upper cervical lymph nodes with corresponding E12 plastinated-embedded sections in the dog. Vet J 2007;174:435-438.

30. Burns GO, Scrivani PV, Thompson MS, et al. Relation between age, body weight, and medial retropharyngeal lymph node size in apparently healthy dogs. Vet Radiol Ultrasound 2008;49:277-281.

31. De Swarte M, Alexander K, Rannou B, et al. Comparison of sonographic features of benign and neoplastic deep lymph nodes in dogs. Vet Radiol Ultrasound 2011;52:451-456.

32. Furukawa M, Dillon JK, Futran ND, et al. The prevalence of lymph node metastases in clinically N0 necks with oral cavity squamous cell carcinoma: is CT good enough for nodal staging? Acta Radiol 2014;55:570-578.

33. Williams LE, Packer RA. Association between lymph node size and metastasis in dogs with oral malignant melanoma: 100 cases (1987-2001). J Am Vet Med Assoc 2003;222:1234-1236.

34. Langenbach A, McManus PM, Hendrick MJ, et al. Sensitivity and specificity of methods of assessing the regional lymph nodes for evidence of metastasis in dogs and cats with solid tumors. J Am Vet Med Assoc 2001;218:1424-1428.

35. Hoang JK, Vanka J, Ludwig BJ, et al. Evaluation of cervical lymph nodes in head and neck cancer with CT and MRI: tips, traps, and a systematic approach. AJR Am J Roentgenol 2013;200:W17-W25.

36. Castelijns JA, vanden Brekel MW. Imaging of lymphadenopathy in the neck. Eur Radiol 2002;12:727-738.

37. Mack MG, Rieger J, Baghi M, et al. Cervical lymph nodes. Eur J Radiol 2008;66:493-500.

38. Kato H, Kanematsu M, Kato Z, et al. Necrotic cervical nodes: usefulness of diffusion-weighted MR imaging in the differentiation of suppurative lymphadenitis from malignancy. Eur J Radiol 2012;82:e28-e35. 